\title{
Hybrid Open Access
}

Bo-Christer Björk, David Solomon

\section{Source}

Bo-Christer Björk and David Solomon. (2014). Developing an Effective Market for Open Access Article Processing Charges (PDF) (Report). Wellcome Trust.

A form of OA where a publisher opens up and makes freely available an individual article in an otherwise subscription based journal based on an author, their institution or funder paying an article processing fee. 